\title{
Phronesis
}

\section{La construction identitaire des éducateurs de jeunes enfants en alternance : ou comment l'usage du construit de reliance participe-t-il de la réorientation de leur projet professionnel en cours de formation?}

\section{The identity building of educators of young children on block-release courses: or how the use of a developed reliance takes part in the reorientation of their professional project in the course of their training}

\section{Marie-Christine Talbot}

\section{Volume 5, numéro 1, 2016}

Les reliances de l'alternance en formation et ses effets sur les processus de construction identitaire des alternants

URI : https://id.erudit.org/iderudit/1037190ar

DOI : https://doi.org/10.7202/1037190ar

Aller au sommaire du numéro

Éditeur(s)

Université de Sherbrooke

ISSN

1925-4873 (numérique)

Découvrir la revue

Citer cet article

Talbot, M.-C. (2016). La construction identitaire des éducateurs de jeunes enfants en alternance : ou comment l'usage du construit de reliance participe-t-il de la réorientation de leur projet professionnel en cours de formation ? Phronesis, 5(1), 4-15. https://doi.org/10.7202/1037190ar

\section{Résumé de l'article}

Si les éléments de contexte, tels que les politiques sociales ou le monde du travail en mutation, viennent en partie éclairer l'émergence d'un nouveau projet professionnel des éducateurs de jeunes enfants en cours de formation, c'est le processus de professionnalisation et de construction identitaire engagé au sein même du processus de formation que nous étudierons et ceci, plus particulièrement en lien avec l'expérience pratique vécue au cours des stages (Wittorski, 2009). Le sujet en formation est, à travers cette situation pratique, dans une posture de réflexion, de construction identitaire par sa rencontre et par sa participation à des communautés de pratiques. Dans sa confrontation à autrui, le sujet met en jeu la réalisation et la continuité de soi. Les émotions, les éléments biographiques sont convoqués eux aussi (Bourgeois et Barbier, 2006), le sujet maintenant, sur le plan identitaire, sa continuité, son unicité à travers le regard d'autrui. Nous chercherons à travers cet article à articuler le processus de professionnalisation et de construction identitaire avec le concept de reliance, développé par Bolle de Bal (2003), dans le cadre de la formation en alternance des éducateurs de jeunes enfants. 


\title{
La construction identitaire des éducateurs de jeunes enfants en alternance : ou comment l'usage du construit de reliance participe-t-il de la réorientation de leur projet professionnel en cours de formation?
}

\author{
Marie-Christine TALBOT
}

Centre de Recherche sur la Formation, CNAM

41, rue Gay-Lussac, 5e étage

75005 Paris

France

mariechristinetalbot@orange.fr

Mots clés : Construction identitaire professionnelle, alternance, reliance, professionnalisation.

Résumé : Si les éléments de contexte, tels que les politiques sociales ou le monde du travail en mutation, viennent en partie éclairer l'émergence d'un nouveau projet professionnel des éducateurs de jeunes enfants en cours de formation, c'est le processus de professionnalisation et de construction identitaire engagé au sein même du processus de formation que nous étudierons et ceci, plus particulièrement en lien avec l'expérience pratique vécue au cours des stages (Wittorski, 2009). Le sujet en formation est, à travers cette situation pratique, dans une posture de réflexion, de construction identitaire par sa rencontre et par sa participation à des communautés de pratiques. Dans sa confrontation à autrui, le sujet met en jeu la réalisation et la continuité de soi. Les émotions, les éléments biographiques sont convoqués eux aussi (Bourgeois et Barbier, 2006), le sujet maintenant, sur le plan identitaire, sa continuité, son unicité à travers le regard d'autrui. Nous chercherons à travers cet article à articuler le processus de professionnalisation et de construction identitaire avec le concept de reliance, développé par Bolle de Bal (2003), dans le cadre de la formation en alternance des éducateurs de jeunes enfants.

Title: The identity building of educators of young children on block-release courses: or how the use of a developed reliance takes part in the reorientation of their professional project in the course of their training.

Keywords : professional identity building, block-release training, "reliance", professionalization.

Abstract: Although the context, such as the social policies or the professional world in mutation, highlights the emergence of a new professional project for educators of young children during their training period, the process of professionalization and the identity building involved during the training period will be more especially studied, in relation to the practical experience gathered during the internships (Wittorski, 2009). This experience awakes in the subject a reflection and a situation of identity building through the encounter and the interactions with practical communities. While confronting with others, the student involves the realization, the self-continuity. Emotions and biographical facts are involved as well (Bourgeois et Barbier, 2006), thus maintaining the subject's continuity and identity uniqueness through the perception of others. In this article, we will relate the process of professionalization and identity building to the concept of reliance, developed by Bolle de Bal (2003), within the scope of block-release training of educators of young children. 


\section{D $\mathrm{R}^{\mathrm{REV}} \mathrm{C}$

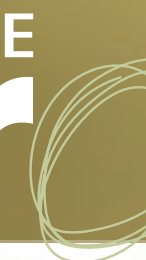

\section{Introduction}

Nous présenterons dans cet article les résultats d'une recherche menée dans le cadre du Master de Formation des adultes dispensé au CNAM.

Dans les formations de travail social, certains étudiants choisissent, à l'issue de la formation d'éducateur de jeunes enfants, d'entreprendre une formation d'éducateur spécialisé. Cette décision peut surprendre dans la mesure où l'entrée en formation est précédée d'épreuves de sélection qui évaluent et qui s'assurent de la connaissance et de la motivation pour ce métier. Cette profession apparaît à la fin du $19^{e}$ siècle avec les propositions des pédagogues de la petite enfance (Fröbel, Pestalozzi et Montessori). Elle se développe aussi avec la préoccupation sociale pour la petite enfance. Cette profession est aujourd'hui définie ainsi :

L'éducateur de jeunes enfants est un travailleur social spécialiste de la petite enfance. Ses fonctions se situent à trois niveaux : éducation, prévention, coordination. Il s'attache à favoriser le développement global et harmonieux des enfants en stimulant leurs potentialités intellectuelles, affectives, artistiques. En créant un environnement riche et motivant, il contribue à leur éveil et à leur apprentissage à la vie sociale (Arrêté du 16 novembre 2005 relatif au Diplôme d'état d'éducateur de jeunes enfants).

Dans ce secteur professionnel fortement féminisé, les dispositifs de modes d'accueil sont porteurs et générateurs d'emplois. Les politiques sociales et familiales sont mises en œuvre en faveur de la petite enfance et des familles. Si les stagiaires se réorientent vers le secteur de l'éducation spécialisée, les arguments en faveur de l'emploi semblent peu significatifs et la définition de la profession précise clairement l'activité et les missions des professionnels.

Alors pour découvrir les éléments qui motive cette réorientation et en comprendre le sens, nous avons choisi de recourir à la méthode qualitative, à partir d'entretiens semi-directifs. Nous avons défini deux échantillons restreints : le premier composé de deux éducatrices de jeunes enfants en formation, le second composé de deux éducatrices de jeunes enfants diplômées en formation d'éducateur spécialisé. L'objectif des entretiens a été de recueillir et d'analyser, à partir de leur discours, les éléments qui les ont conduites à entreprendre la formation d'éducatrice de jeunes enfants, à conforter ou à réorienter leur projet professionnel initial.

À partir de l'analyse des entretiens, nous avons élaboré une grille en retenant les catégories suivantes : le parcours avant l'entrée en formation, l'origine du projet professionnel, le rôle des stages dans la construction identitaire professionnelle, la représentation du métier d'éducateur de jeunes enfants et celle d'éducateur spécialisé, le projet professionnel envisagé après la formation et pour les stagiaires en formation d'éducateur spécialisé, le passage d'une formation à l'autre.

Ici nous nous centrerons plus particulièrement sur les éléments recueillis autour des stages. Nous chercherons à faire dialoguer le concept de reliance de Bolle de Bal (2003) avec la question des processus de professionnalisation et de construction identitaire.

\section{Professionnalisation, construction identitaire, reliance}

Le cadre théorique se réfère aux concepts de professionnalisation (Wittorski, 2009), de construction identitaire (Dubar, 2000, Barbier et Bourgeois 2006) et de reliance (Bolle de Bal, 2003). Ces apports théoriques, complétés par le concours d'autres auteurs, éclaireront la réorientation des éducateurs de jeunes enfants en formation. Nous chercherons à considérer les liens entre, d'une part, les processus de professionnalisation et de construction identitaire et, d'autres part, le concept de reliance. 


\subsection{Professionnalisation et construction identitaire : deux processus en interaction}

L'évolution des politiques sociales et économiques en Europe conduit à d'importantes transformations en termes d'organisation et de management au sein des entreprises. Cet élément de contexte induit de nouvelles attentes du monde du travail, qui se répercutent au niveau des salariés, sommés de s'adapter aux nouvelles exigences d'organisation. Wittorski, en référence à Leboterf (2011), soulève la question de la responsabilité et de la compétence. II considère qu'il s'agit d'un nouveau sujet contemporain et commun à l'ensemble des organisations. En effet, pour chaque salarié, il est attendu qu'il sache : organiser son travail, conduire son propre parcours professionnel, recourir aux dispositifs de formation tout au long de la vie. La transformation sociale agit sur l'organisation du travail et par conséquent sur l'adaptation des formations. La transformation sociale, induite par les nouvelles formes d'organisation socio-économique, agit aussi sur les processus de construction identitaire (Dubar, 2000, Barbier \& Bourgeois, 2006). Les étudiants en travail social entrent en formation dans ce contexte particulier.

La formation, au delà du contexte dans lequel elle s'exerce, est aussi un processus qui entraîne le sujet dans une négociation identitaire entre d'une part, son environnement personnel et social et, d'autre part, le monde professionnel auquel il se destine. Ses valeurs, ses intérêts et son projet vont être interrogés. L'identité réflexive (Dubar, 2000) prend une place importante dans la manière dont les individus vont s'orienter. Cette dimension réflexive conduit le sujet à s'adapter aux transformations. Pour cela, il doit se penser et se repenser. L'identité réflexive agit aussi sur le processus de professionnalisation et sur le processus de développement personnel du sujet en formation.

En ce sens, la problématique de la professionnalisation est reliée à celle de la construction identitaire (Wittorski, 2005). En effet, le sujet en formation est à la fois engagé dans son projet professionnel et dans une négociation identitaire entre les valeurs et les intérêts qui fondent en partie son identité personnelle. L'identité professionnelle en construction, ici celle des éducateurs de jeunes enfants, est un processus complexe dans lequel s'articulent et se mettent en tension les dimensions biographique, relationnelle et intégrative du professionnel en devenir (Perez-Roux, 2011).

La négociation identitaire met en jeu l'identité attribuée, reconnue par un tiers pour le sujet. Elle met aussi en jeu l'identité narrative (Dubar, 2000), qui inscrit le sujet dans un contexte familial, géographique, historique qui lui a transmis des manières de penser, d'agir et de se conduire. L'identité narrative s'adresse à autrui. Elle participe à la construction du sujet. Le sujet se présente à autrui pour autrui. Mais il se présente aussi à autrui, pour lui, par la présentation de l'image de soi. Lorsque le sujet intègre un centre de formation ou un lieu de stage, il découvre de nouveaux environnements. II prend connaissance par cette intégration de ses éléments identitaires.

Cela conduit le sujet à réaliser des choix de continuité, de rupture ou encore de transaction pour lier à la fois le projet de formation et la continuité de soi. Ce processus produit pour chaque sujet des logiques différenciées pouvant aller du refus du changement, à la transformation partielle ou à la réorientation du projet professionnel (Perez-Roux, 2012). Cela nécessite une adaptation du sujet. L'individu possède cette capacité de pouvoir, de vouloir ou de devoir s'adapter au milieu dans lequel il vit (Astier, 2008). Le sujet et le groupe professionnel sont engagés simultanément dans ce processus d'adaptation et de professionnalisation. Ils partagent en effet cette préoccupation commune de préservation et de continuité visant à donner sens et cohérence à leur activité. Ainsi, lors de la rencontre avec un nouvel environnement, lors des stages notamment, les sujets en formation sont conduits à modifier certaines de leurs valeurs, à s'approprier de nouveaux rôles conduisant à la transformation de l'image de soi (Perez-Roux, 2012).

Dans le processus formatif, l'identité est en mouvement mais aussi en tension. Les composantes de l'identité du sujet sont en tension, car la formation conduit à un déséquilibre par l'interaction du je et du nous (Dubar, 2007). Un environnement nouveau vient interroger ce qui a été transmis au sujet jusqu'à présent et incorporé par lui. Le sujet, dans ce processus de construction identitaire (Bourgeois, 2006), va chercher un nouvel équilibre, dans la préoccupation de la continuité de soi et dans la préservation de sa cohérence.

Le processus identitaire fait aussi appel à la dimension du langage (Barbier 2006). Cette dimension exprime la question identitaire. Elle révèle la réciprocité entre le sujet et autrui. Elle montre aussi l'importance de l'activité et des émotions dans ce processus de transformation. Le langage communique ces différentes dimensions et ces tensions constitutives de l'identité en construction. Ce processus peut-être source de plaisir, mais aussi de souffrance. Il est dans le champ du sensible et dans le champ de la communication. Les émotions et les relations interpersonnelles agissent (Bourgeois, 2006) dans le processus de construction identitaire. L'individu cherche à maintenir sa continuité. Il défend son unicité dans et par le regard d'autrui. Les trois instances au travail dans la construction identitaire sont alors : le Soi actuel, le Soi idéal et le Soi normatif. La formation, comme projet de soi pour soi, est aussi un projet pour autrui (Kaddouri, 2006). En effet, le sujet est soumis au regard et aux attentes d'autrui par l'environnement professionnel et personnel. Cela met en lumière l'aspect complexe du sujet en action, 
dans un environnement agissant sur lui, lui-même agissant, à son tour, sur l'environnement. Ainsi, le processus de formation met en jeu pour le sujet (Dubar, 2000) la double dimension de l'identité : personnelle et sociale.

Dans l'espace professionnel sont produites des représentations communes qui participent à la production de représentations collectives. L'individu, le professionnel ou le stagiaire en formation, adhèrent à ces images groupales qui lui apportent un bénéfice individuel (Barbier, 2006). Ce bénéfice est celui de la reconnaissance de soi dans ces images groupales. II traduit son projet d'appartenance à un groupe. Mais le sujet peut aussi ne pas se reconnaître dans le groupe et chercher à s'en dégager. L'identité se construit par l'altérité (Sebastian et de Villers, 2006). Mais cette cette altérité dépasse le groupe d'appartenance. Toutefois, ce dernier est porteur d'une dimension plus symbolique du fait des éléments incorporés par les individus dans lesquels autrui est présent. Ainsi, les stagiaires, porteurs d'éléments du groupe qu'ils ont incorporés, transmis par leur environnement personnel, s'approprient au cours des stages de nouvelles images véhiculées par les communautés de pratiques notamment par le langage et par les activités réalisées (Bourgeois, 2006).

Dans le cadre d'une formation, les apprenants mettent en jeu les composantes de leur identité. Le processus de construction identitaire, induit par la formation, conduit le sujet à chercher un équilibre entre son projet de formation, les images professionnelles projetées émanant du groupe professionnel et celles révélées lors des stages dans le cadre par exemple des communautés de pratique. Dans cette perspective, la formation devient une offre de significations, autrement dit, une offre de sens attribuée à l'apprentissage et qui participe à la construction de soi (Barbier, 2006). Cette offre de significations est indicative des rôles sociaux, des attendus de la conduite professionnelle du sujet et donc de la conduite de son soi professionnel.

La tension identitaire, se nouant à ce moment pour le sujet, se traduit par des conflits sociocognitifs (Bourgeois, 2006). Elle ouvre la voie à la délibération sur soi dans un dialogue avec les nombreux «moi » révélés par la formation (Barbier, 2006). Cette délibération conduit (Neuville, 2006), lors de la formation, à faire des choix et donc à renoncer à d'autres identités possibles.

Nous pouvons alors considérer la formation comme un événement et comme un moment spécifique de la vie du sujet participant à sa propre construction identitaire. Le sujet, confronté à ses interrogations, s'interrogeant sur son engagement, bousculé par ce processus de construction-transformation identitaire va alors entreprendre de se lier, de se relier ou de se dé-lier à autrui mais aussi à des valeurs et à des pratiques. Le sujet cherche ainsi à donner sens à ses choix et à élaborer sa continuité, voire sa cohérence identitaire. C'est en ce sens que le concept de reliance participe de la professionnalisation et de la construction identitaire des sujets en formation.

\subsection{L'acte de reliance au regard des processus de professionnalisation et de construction identitaire}

Dans ce processus de formation et de transformation, le concept de reliance, défini par Bolle de Bal, nous intéresse (2003). II s'agit pour Bolle de bal d'un acte ou d'un état concernant au moins une personne humaine. L'auteur identifie des expressions ou des termes synonymes au mot relier : créer ou recréer des liens, établir une liaison entre d'une part, une personne et, d'autre part, soit un système ou un sous-système dont la personne fait partie. Nous pouvons considérer que les différentes dimensions de l'identité, mises en jeu dans le processus de construction-transformation identitaire et dans les processus de professionnalisation, sont des systèmes dont les sous-systèmes ou parties cherchent à se relier afin d'établir une liaison cohérente au regard des valeurs, des intérêts et du projet du sujet.

Le concept de reliance porte deux significations : la première exprime l'acte de reliance (Bolle de Bal, 2003) c'est-à-dire la reliance mise en oeuvre et réalisée. La seconde exprime l'état de reliance, autrement dit, la reliance vécue.

La reliance recouvre plusieurs dimensions dont celle de la reliance sociale définie comme une production de rapports sociaux médiatisés, autrement dit, une médiatisation des liens sociaux (Bolle de Bal, 2003). À travers les situations de formation, chaque stagiaire médiatise des liens sociaux avec différents acteurs : des référents de stage, des formateurs, des collègues mais aussi le public dans l'exercice de l'activité. Ainsi l'acte de reliance est à l'œuvre dans l'adaptation du sujet au contexte. Mais cet acte de reliance met en jeu l'altérité dans la mesure où il crée les conditions de la rencontre de l'autre dans sa singularité. II s'agit alors pour le sujet d'entreprendre un travail de reliance pour connaître, reconnaître et être reconnu par autrui. L'autre peut être le groupe professionnel, une commaunauté de pratique ou un membre du groupe. Par le prisme de l'altérité, nous pouvons définir et comprendre l'acte de reliance comme un processus de continuité de soi dans une présentation de soi à autrui afin de créer des reliances. Il s'agit ici d'une reliance de soi à autrui et d'une reliance de soi à soi.

La reliance, par l'activité cognitive, émotive et langagière qu'elle induit, participe du processus de professionnalisation et du processus de construction-transformation identitaire du sujet en formation. Dans cette perspective, la reliance à autrui interagit avec la dimension personnelle des sujets en relilance. Si la construction identitaire est en partie déterminée et influencée 
par autrui, elle n'en reste pas moins un débat de soi avec soi, sur soi, pour soi, ce débat étant suscité par les reliances à autrui. Le concept de reliance conduit donc à interroger la dimension intrapsychique du sujet, concerné par la conduite de reliance à soi, autrement dit de reliances entre le sujet et les différentes composantes de son identité (Bolle de Bal, 2003).

À travers l'expérience de formation, le sujet fait acte de reliance. Pour cela, il s'appuie sur des systèmes médiateurs que sont le langage et la participation à des communautés de pratiques. II cherche à articuler ce qui est séparé et à relier ce qui est disjoint (Le Moigne, 2008). Cela peut le conduire à un engagement, à un retrait partiel du groupe d'appartennance socio-professionnel ou de la communauté de pratiques ou à une réorientation. Nous voyons alors que le concept de reliance contient aussi les dimensions de liance et déliance constitutives de la production de l'acte de reliance (Bolle de Bal, 2003,).

\section{3 La réorientation professionnelle des éducateurs de jeunes enfants : trois hypothèses}

Pour comprendre ce qui motive la réorientation des éducateurs de jeunes enfants vers la profession d'éducateur spécialisé, tout ceci se déroulant au cours où à l'issue de la formation, nous explorerons trois hypothèses :

\section{L'expérience pratique, comme facteur d'interrogation du projet professionnel initial}

L'expérience pratique est au cœur de la construction identitaire professionnelle. En quoi l'expérience de stage est, ou non, un facteur d'interrogation voire de réinterprétation du projet professionnel formulé lors de l'entrée en formation, qu'il s'agisse de s'orienter vers le champ de la petite enfance ou vers celui de l'éducation spécialisée?

Dans les dispositifs de formation en alternance, la dimension de l'action du sujet interroge et participe de sa construction identitaire (Wittorski, 2005). Les différentes formes d'identité, réflexive et narrative, sont particulièrement sollicitées. Au cours de leur formation pratique notamment, les sujets en formation rencontrent, participent et s'intègrent aux communautés de pratiques au sein desquelles ils exerceront leur métier. Ces communautés de pratiques sont celles avec lesquelles les sujets se relient par un processus de professionnalisation et de développement professionnel et par lesquelles ils élaborent progressivement leur identité professionnelle.

\section{Petite enfance ou éducation spécialisée : projet professionnel et valorisation de soi}

La représentation des champs professionnels est variable pour chaque sujet en formation et cela est fonction de son environnement, de son expérience et de ses aspirations. La petite enfance ou l'éducation spécialisée peuvent représenter pour ces futures professionnelles un secteur plus ou moins valorisé et valorisant selon qu'il permet ou non la réalisation de leurs aspirations et de leurs idéaux. L'expérience pratique crée une tension entre le soi et l'idéal de soi (Dubar, 2007). Elle fait également écho aux éléments vécus et hérités de l'enfance, éléments constitutifs de l'identité personnelle. Cela conduit le sujet à interroger l'image de soi pour autrui et l'image de soi pour le sujet lui-même. C'est ici la question de la comparaison et de l'évaluation des sujets (Barbier, 2006) à partir de leurs propres grilles d'analyse d'une réalité et de leur réalité (Cohen-Scali \& Moliner, 2008). Que perçoivent-ils comme source de valorisation? La situation de formation conduit alors à une délibération sur le «soi professionnel». La notion de reliance se manifeste ici dans un processus de négociation sur soi, à travers les nouvelles offres de sens d'une réalité. Le sujet a l'opportunité de nouveaux liens qu'il cherchera, ou non, à s'approprier et à développer.

\section{Transformation de soi et continuité de soi}

Dans le processus de construction identitaire professionnelle, le sujet en formation cherche à se développer et à se préserver. II tend à percevoir sa propre continuité et une forme de congruence. En effet il s'agit pour le sujet, au regard de son activité, de ses aspirations et de son histoire, de conserver une cohérence entre ce qu'il est ou perçoit être et ce à quoi il aspire (Bourgeois, 2006). Dans cette hypothèse, il est nécessaire de prendre en compte la dimension de l'histoire personnelle constitutive de la trajectoire de l'individu. La dimension des émotions et la mise en jeu de celles-ci dans les relations interpersonnelles, la dimension de l'altérité dans la construction identitaire professionnelle invitent à définir le concept de reliance à travers l'étude des interactions liées ou construites par l'alternance.

\section{Construction identitaire des éducateurs de jeunes enfants : entre continuité et réorientation}

Nous allons présenter une partie des verbatim des entretiens. Nous allons tout d'abord regrouper les éléments significatifs issus des entretiens des éducateurs de jeunes enfants en formation. Puis nous présenterons ceux issus des entretiens des éducatrices de jeunes enfants inscrites en formation d'éducateur spécialisé. 


\subsection{Continuité de soi et confirmation du projet professionnel}

Chloé et Caroline rapportent leur formation d'éducateur de jeunes enfants à travers leur parcours de stage.

\section{Le parcours de Chloé}

Chloé se trouve être en fin de formation d'éducatrice de jeunes enfants. Elle présente ses différents stages. À travers l'analyse de ses dires, nous allons chercher à éclairer le processus de construction et de négociation identitaire.

Son premier stage se déroule en crèche collective hospitalière auprès de bébés. Cette situation est représentative des lieux d'exercice professionnel des éducateurs de jeunes enfants. Chloé rencontre des professionnelles investies, mais fatiguées, ditelle, par leurs conditions de travail dans l'institution. Chloé rapporte: "la crèche, c'est un héritage hygiéniste et du coup moi, je trouvais ça intéressant d'aller questionner ça ». Elle relie cette expérience à sa connaissance de l'histoire du métier. Elle crée du matériel pour les bébés et observe notamment la relation mère-enfant. Elle dit s'émerveiller et considère que c'est vraiment le métier qu'elle veut faire. Ce premier stage, malgré des difficultés liées à l'organisation et tout particulièrement aux rythmes de travail, confirme son projet de formation.

Son second stage se situe en école maternelle. La directrice de l'école mobilise une pédagogie innovante fondée sur l'usage du bilinguisme. Même si pour Chloé, ce stage ne fait pas suffisamment appel à des savoirs théoriques, il convoque, selon elle, la question des savoir-être : « là où j'ai trouvé ça hyper chouette dans son projet aussi, c'est parce qu'elle parle beaucoup de savoir-être. Et elle forme du coup ses équipes là-dessus, je trouve ça vraiment très intéressant. » Ici, la dimension théorique même insuffisante à ses yeux, est dépassée par la dimension relationnelle avec l'équipe et l'aspect innovant du projet pédagogique.

Chloé explique au cours de l'entretien qu'elle a été sensibilisée à l'Art dans sa famille, ce qui motive son orientation vers une structure située dans une organisation culturelle et pouvant accueillir des enfants de moins de six ans. Ce type d'organisation s'inspire de l'initiative de Françoise Dolto : la maison verte. C'est le troisième stage de Chloé. Les professionnels sont psychologues ou psychanalystes. L'éducatrice de jeunes enfants est directrice de la structure. Elle est chargée du partenariat avec différentes organisations : des crèches, un institut médico-éducatif et une maison de quartier. Chloé dit : "Voilà, j'avais cette affinité avec l'Art et donc ça me paraissait juste en plein dans le 1000 de faire mon stage dans un lieu d'accueil parentsenfants, ce sont des enfants de moins de six ans qui viennent accompagnés de leurs parents ». Ce troisème stage rassemble son double intérêt pour l'Art et la petite enfance. Elle relie, par le langage, son héritage identitaire auquel elle adhère et son projet professionnel en recherche de continuité et de cohérence. Cette expérience, dans son parcours de formation, confirme son intérêt pour un travail en équipe, pour un projet innovant et pour un travail de médiation dans la relation enfant-parent : «C'est vraiment la maison verte de Françoise Dolto, oncle tante, papa, le grand-père et voilà. Les accueillantes sont psy, [...] c'est un peu à l'encontre de tout ce qui se fait dans la petite enfance c'est-à-dire que les psychologues sont en majorité, vous êtes la seule éducatrice de jeunes enfants sur le terrain, la directrice de la maison des petits est éducatrice de jeunes enfants, elle n'est pas vraiment sur le terrain [...], la responsable c'est quelqu'un... c'est pour moi la responsable, c'était une super belle rencontre et c'était ma référente de stage ». Dans le cadre de ce stage, elle rencontre une figure professionnelle à laquelle elle s'identifie car elle exprime une représentation du travail dans le secteur de la petite enfance basée sur le partenariat et sur une communauté de pratiques. Cela lui permet d'affirmer et de développer sa conception du métier à partir de cette figure centrale d'éducatrice de jeunes enfants agissant au sein d'une structure innovante.

Elle choisit ensuite un autre stage, cette fois en ludothèque. Elle considère le jeu comme une spécificité des éducateurs de jeunes enfants. Elle porte un regard critique sur l'accueil des familles au sein de la ludothèque : « après euh, les ludothécaires ça m'a posé question dans le sens où demain si vous voulez vous appeler ludothécaire, y'a pas de formation, venant d'un lieu d'accueil parents enfants, je voyais tout ce qui se passait dans la relation, et elle passait peut-être à côté de plein de choses, [...]. Du coup là enfin moi je me suis vraiment positionnée en tant qu'éducatrice de jeunes enfants ». Ce stage, répondant partiellement à ses attentes, agit à ce moment comme un renforcement de son choix professionnel et comme une affirmation des valeurs portées par ce métier dont elle se fait le porte-parole. Dans son discours, Chloé relie et valorise son expérience de stage à travers ce qui est essentielle pour elle et qui constitue le fil conducteur de sa pratique : la relation avec les familles.

Elle terminera sa formation pratique dans une école maternelle Montessori afin, dit-elle, de se faire plaisir et pour approfondir la pédagogie. La pédagogie, selon ses propos, nourrit sa réflexion sur le lien adulte-enfant. 
Ainsi tout au long de son parcours de formation, Chloé tisse un lien étroit entre ses différentes découvertes et ses objets d'intérêts. Ces expériences pratiques viennent confirmer son choix et préciser ses intentions. Au-delà du secteur de la petite enfance, il s'agit pour elle de travailler autour de la relation parent-enfant.

\section{Le parcours de Caroline}

Caroline, éducatrice de jeunes enfants, est en fin de seconde année de formation. Elle dit, au début de l'entretien, que les stages sont déterminés par le centre de formation à travers des passages obligatoires : crèches, ludothèques et lieux spécialisés.

Elle commencera donc par décrire un stage d'observation auprès de jeunes enfants en situation de handicap dans un institut d'éducation motrice: «Pour moi, c'était un stage très difficile, d'autant plus que le handicap, ça n'est pas forcément ma tasse de thé à la base ». Sa remarque exprime une expérience difficile et vécue sur le registre de la contrainte. Malgré le jeune âge du public il y a pour elle une impossibilité à se relier, à se reconnaître dans cette expérience professionnelle.

Caroline effectue ensuite un stage dans une crèche collective dont la particularité est d'accueillir des enfants déficients auditifs. Après avec cette expérience de stage, elle dit, à propos de son projet professionnel : " clairement pas du tout en crèche collective «. Ici, ce n'est pas le handicap qui est perçu comme complexe ou difficile, mais la forme de prise en charge à savoir la crèche collective.

Ensuite, elle choisit un troisième stage, situé en centre maternel. Ce stage réunit toutes les problématiques qui l'intéressent. Elle évoque particulièrement la dimension de la parentalité : " parce que c'est un objectif principal du travail de base de l'éducatrice de jeunes enfants ». Elle ajoute : "J'aime les défis et les choses un peu compliquées, le travail est riche et différent d'une crèche classique ». Elle poursuit : « les enfants ont besoin de nous [...] il y a vraiment un accompagnement individualisé pour le coup ce qu'on ne retrouve pas vraiment en crèche collective et après, en fait tout prend une ampleur beaucoup plus importante ».

À l'analyse de ces verbatims, nous pouvons identifier plusieurs éléments. Son choix professionnel est motivé par le travail spécifique autour de la parentalité. Elle affirme sa volonté de travailler en dehors des crèches collectives. Elle argumente sa position par le manque d'accompagnement individualisé. Elle établit un lien avec son parcours universitaire antérieur : « je n'ai pas fait cinq années de psycho pour rien! Donc ben forcément moi je sais j'ai fait de la thérapie etc. parce que en psycho c'est indispensable [...] dans le travail de l'éducatrice de jeunes enfants, c'est aussi indispensable parce que on est confronté à des enfants, et à des mamans qui nous renvoient des choses de notre histoire personnelle. Et là il faut vraiment avoir conscience, je sais que c'est guidé et moi je sais que mes choix je sais que c'est guidé par mon histoire personnelle ». Dans cette affirmation forte, nous percevons l'importance donnée par Caroline à la cohérence de son projet professionnel à la fois dans une perspective comptable (elle a effectué cinq années de psychologie), mais aussi personnelle. Elle relie, dans cette posture réflexive, son expérience personnelle (scolaire et affective) à ses attentes professionnelles. Elle explique qu'au centre maternel, elle a rencontré une chef de service impressionnante. Elle envisage dès lors une évolution future vers le management.

Au cours de sa troisième année de formation, Caroline devra réaliser deux stages. Elle se projette en ludothèque. Elle souhaite se perfectionner par rapport au jeu. Elle cherche à établir un lien avec les parents dans le cadre d'une expérience professionnelle en service hospitalier (service périnatal) ou en pouponnière. Nous voyons ici une orientation liée au centre de formation et une projection argumentée en lien avec sa conception du métier.

\subsection{Réorientation du projet professionnel vers l'éducation spécialisée}

Charlotte et Claire sont en formation d'éducateur spécialisé à la suite de leur formation d'éducatrice de jeunes enfants. Elles reviennent sur leur parcours de formation.

\section{Le parcours de Charlotte}

Charlotte a réalisé son premier stage en crèche. De cette première expérience pratique, elle en souligne le caractère répétitif : « insupportable, le premier stage je l'ai fait en crèche, ça m'a paru tellement long ça a duré deux mois, [...] j'ai le sentiment que ça n'était pas fait pour moi et elle ajoute : j'en ai fait qu'un seul en crèche, parce que j'étais obligée, les formateurs m'ont obligé et donc voilà ». Ce premier stage développe, dit-elle, un sentiment d'inutilité qu'elle résume ainsi : " en crèche, je me sentais inutile ». Dès le début de formation, Charlotte déclare écarter cette organisation de travail. 
Elle s'oriente ensuite vers un stage dans un institut médico-éducatif auprès d'enfants en situation de handicap (déficience intellectuelle). Charlotte n'est pas très à l'aise avec ce public, mais elle y trouve un intérêt : "le stage de l'IME du coup à ce moment-là dans la formation c'est le premier stage où j'ai côtoyé un peu les difficultés familiales en plus du handicap il y avait aussi toute la problématique autour des parents... c'est ce côté-là qui m'a après tout fait un peu rester dans le milieu des difficultés sociales...c'est pour ça que j'ai continué ». À ce moment, son intérêt pour les difficultés familiales justifie et renforce son intérêt pour ce stage qui se situe dans le champ du handicap.

Les stages suivants se dérouleront en protection de l'enfance, dans une maison à caractère social et dans un service de placement familial où l'équipe lui laisse une place de professionnelle à part entière : « je suis très intégrée à l'équipe...j'y allais c'était le bonheur, j'y ai passé deux journées jamais pareilles, en fait toutes les caractéristiques qu'il me fallait.» Ici, elle se sent appartenir à cette communauté de pratiques. Elle se reconnaît et est reconnue. Les formes de travail la satisfont. L'activité est diversifiée et non routinière. Elle engage des relations avec de nombreux partenaires extérieurs : les personnels de l'école et les travailleurs sociaux de différents services.

Elle pose un regard critique sur le rôle des éducateurs de jeunes enfants dans ce service : « elles faisaient les visites, mais centrées sur l'enfant, c'était elles qui s'occupaient de tout ce qui est jouet, elles organisaient les salles, c'est elles qui organisaient tout ce côté-là le matériel autour de l'enfant dans une visite médiatisée ».

Puis, elle compare cette activité à celle des éducateurs spécialisés : «ils sont plus à parler de la situation voilà,... avec les parents «comment vous allez...» tout ça alors que l'éducatrice de jeunes enfants elle, c'était vraiment centré sur l'enfant ...c'est elles qui m'ont appris le métier d'éducatrice de jeunes enfants, mais en même temps je voyais le métier d'éducateur spécialisé et je ne pouvais pas y accéder et du coup c'est quelque chose qui... qui m'a... et en même temps je me suis dit c'est vraiment ça que je veux faire que je sois le EJE ou ES, mais en tant qu'EJE je ne pourrai pas ». À travers cette comparaison, elle opère des choix. Elle déclare préfèrer des activités. Elle en élimine d'autres. Cela participe du processus de construction identitaire et cela semble réduire la tension identitaire. Elle explicite alors sa réorientation. Les aspects du travail d'éducatrice de jeunes enfants sont, dit-elle, limités. C'est l'argument qui la conduit à entreprendre la formation d'éducatrice spécialisée. Même si cette idée avait déjà émergé lors du premier stage en institut médico-éducatif, c'est l'expérience dans le secteur de la protection de l'enfance qui sera décisive pour sa réorientation vers la formation d'éducateur spécialisé. Dans son discours, elle exprime le désir de dépasser le cadre de l'activité des éducatrices de jeunes enfants, qu'elle perçoit comme un travail prescrit, encadré dans une organisation stricte de l'espace, du matériel et de l'enfant. Elle aspire à une activité professionnelle où elle puisse se sentir utile, ce qui lui permettrait de se réaliser et où les modalités d'intervention sont ouvertes dans une clooaboration et une coopération avec des partenaires internes et externes.

\section{Le parcours de Claire}

Au cours de sa formation d'éducatrice de jeunes enfants, Claire réalise deux stages auprès de jeunes enfants : I'un en crèche, l'autre en halte d'enfants» Elle déclare être déçue de ces deux stages. "Les journées me paraissaient longues, très longues, finalement je trouvais qu'à part, parler avec les collègues, on ne faisait pas grand-chose je trouvais que derrière l'action ça manquait beaucoup de réflexion ».

Elle dit éprouver de l'ennui dans ces structures de la petite enfance. Elle étouffe, dit-elle. Elle a l'impression d'un quotidien qu'il faut remplir absolument par des activités sans forcément que cellesci soient réfléchies: "c'est plaqué, ce qui me gênait et puis euh les professionnels était un peu, je les trouvais un peu, enfermées, ce, ce n'est pas vraiment le monde, je crois qu'elles avaient tellement finalement de connaissances que ça les empêchaient d'ouvrir et de voir que un enfant ce n'est pas forcément écrit dans les livres et qu'il y a plein de choses et que l'on peut, l'enfant il peut évoluer différemment de ce qu'on a pu lire chez Montessori voilà ». Cette expérience révèle chez Claire une discordance entre la représentation du métier d'éducatrice de jeunes enfants (ici la manière d'agir dans la vie quotidienne auprès de l'enfant) et celle des professionnelles qu'elle rencontre. Nous pouvons dire qu'elle ne se reconnaît pas dans l'offre de sens qui lui est faite, au regard de ses valeurs et de ses intérêts personnels (Barbier, 2006).

Lorsqu'elle effectue un autre stage, cette fois auprès d'enfants en protection de l'enfance, elle dit : « oui, la vraiment ça m'a ouvert, ouvert les yeux sur le travail que je voulais faire... Tant auprès des enfants, auprès des familles, cette question de parentalité... Là, je me suis dit, c'est vraiment où je veux travailler, je touche vraiment du doigt ce qui m'intéresse, ce qui me porte [...] pour aller travailler quoi vraiment, avec, et puis avec des gens qui avaient des convictions, des connaissances sur la protection enfance, sur la parentalité, sur les enfants, des connaissances pointues avec des choses vraiment ». Cette expérience agit comme une révélation. De plus, elle lui ouvre de nouveaux horizons. "C'est la découverte d'un monde ça m'a ouvert tout un univers ». Ici nous comprenons combien pour Claire, la motivation et l'affirmation d'un choix professionnel permettent de 
donner cohérence et équilibre au service d'un projet de développement personnel et professionnel. Le dispositif de formation en alternance offre ici des expériences pratiques favorisant ces allers retours et ces déplacements dans les différents espaces de formation engageant ainsi le sujet en formation vers une délibération sur soi.

Le stage suivant se déroule en centre maternel. Claire dit : « j'ai découvert beaucoup de choses, finalement, en crèche, on est peu amené à travailler avec les adultes, on est peu amené à travailler sur les problématiques des parents. Là, au centre maternel il fallait travailler en même temps sur les problématiques des parents en même temps sur les problématiques des enfants, qui sont, somme toute, très régulièrement liées,... Tout ce travail sur la parentalité c'est ce qui m'a passionné pour lequel je n'ai pas cessé tout au long de ma formation et même actuellement de me questionner et de réfléchir ». Dans ce passage, Claire relie les deux expériences de son parcours professionnel. Elle nous donne les arguments qui motivent sa réorientation et son souhait de travailler avec un public adulte, notamment des adultes en difficultés. Dans son discours réflexif, elle relie différents éléments afin d'élaborer cette continuité, nécessaire au sujet, et visant à donner sens et cohérence à son parcours. Elle argumente encore sa réorientation : " puis voilà, on pouvait aller à l'extérieur rencontrer d'autres professionnels, je trouvais vraiment qu'il y avait une richesse en termes d'inter professionnalité, en termes de partenariat avec la ville avec l'extérieur qui permettait vraiment de nourrir notre travail de réflexion et qui nous permettait de ne pas de ne pas nous enfermer dans nos propres représentations dans nos propres idées ». Ce stage renforce son choix. Progressivement, la décision de devenir éducatrice spécialisée est pour elle une évidence. Elle décide alors d'entreprendre cette formation.

\section{Processus de reliance et construction identitaire professionnelle : analyse et premiers résultats}

Dans la partie suivante, nous allons analyser les éléments recueillis pour chaque échantillon.

\subsection{Chloé et Caroline : élaboration et affirmation du projet professionnel}

Chloé relie son activité à des éléments concrets ayant particulièrement compté pour sa formation : le jeu, le matériel pédagogique, l'importance de l'Art, intérêt qu'elle attribue à son environnement familial. Le lieu d'accueil enfants-parents, du type de la maison verte, souligne la continuité de son intérêt pour la petite enfance et notamment pour une pédagogie et un mode d'accueil vécu comme différent. Le lien et la relation entre les enfants comme avec les parents sont, pour Chloé, centraux. À plusieurs reprises, elle nomme des pédagogues de référence dans le champ de la petite enfance, comme Maria Montessori ou Françoise Dolto.

De la même façon Caroline porte son intérêt vers des lieux où les enfants ont semble-t-il besoin d'elle. Son orientation est guidée par des raisons personnelles. Cela est au cœur des choix qu'elle opère et qui oriente son processus de professionnalisation. La professionnalisation (Astier 2008) est définie en fonction des points de vue des acteurs et de leurs enjeux. Chloé veut participer au monde professionnel. Elle souhaite intervenir dans ce petit espace de la relation parent-enfant. Caroline, quant à elle, relie ses deux expériences de formation présente et passée (formation en psychologie). Toutes deux délibèrent sur elles-mêmes et construisent, par le récit de leurs stages, la cohérence entre les différents éléments de leur construction identitaire professionnelle.

Les stages viennent donner sens à l'orientation professionnelle de ces deux jeunes femmes. Le processus de construction -transformation identitaire (Wittorski, 2006) se trouve renforcé par les images véhiculées par les professionnelles rencontrées et par leur activité qui se veut être en cohérence avec leurs propres représentations du métier. Cela favorise la congruence et la continuité de soi (Barbier, 2006) sans réinterroger l'orientation même du métier (Dubar, 2000). Les formes identitaires sont inséparables des rapports sociaux. L'identité professionnelle se construit dans l'altérité et dans l'interaction d'un Je et d'un Nous. Dans ce processus, les deux jeunes femmes se relient à autrui c'est-à-dire aux communautés de pratiques auxquelles elles souhaitent appartenir.

Comme l'explique Bourgeois, la construction identitaire s'élabore à partir de l'action, à partir de la rencontre et de la participation à des communautés de pratiques (2006). Dans les entretiens analysés, chacune rapporte sa relation aux professionnels et à leur manière de penser et d'agir. Le groupe de pairs fait référence (Dubar, 2007). Pour chacune d'entre elles, il y a adhésion à des figures professionnelles. Pour Chloé, ce sera la directrice-éducatrice de jeunes enfants du lieu d'accueil parents enfants. Pour Caroline, se sera, au centre maternel, la cadre du service. Elles font ainsi référence à des images groupales qui induisent des affiliations identitaires. Nous les voyons, au fil de leur pensée et de leur narration, tisser les liens, retenir ce qui fait sens pour elles, incorporer des éléments du récit, mais aussi se délier de certaines expériences ou de formes de travail de leurs pairs lorsque celles-ci sont en opposition avec leurs véritables aspirations. 
Elles valorisent les professionnels du groupe de référence auquel elles se destinent. Par leur adhésion et par la valorisation des pratiques professionnelles identifiées, elles bénéficient, elles-mêmes, d'une certaine reconnaissance puisqu'elles s'y associent pleinement (Barbier, 2006). Comme l'analyse Bourgeois (2013), autrui joue un rôle essentiel de reconnaissance du sujet, mais aussi d'identification et de repère dans le processus de construction identitaire.

Dans les entretiens, nous notons une confirmation de l'orientation vers le champ de la petite enfance. Les stages réalisés en alternance structurent la logique de «réflexion-action» (Wittorski, 2009). En effet, la dimension réflexive sur l'activité occupe une place importante dans l'alternance et concourt à l'appropriation des modèles culturels, des professionnels rencontrés. Dans le processus identitaire en construction, les sujets cherchent alors à comparer, à trouver des images afin de se situer, de se construire et de se continuer en cohérence (Barbier, 2006).

L'expérience des stages vient renforcer l'orientation vers le champ de la petite enfance, mais à l'intérieur de ce champ elles choisissent, délimitent leur espace professionnel.

Si la formation met en tension les différentes dimensions de l'identité personnelle et professionnelle, c'est par le tissage de liens nouveaux, la consolidation de certains ou encore l'abandon d'autres, que chaque sujet recherche son équilibre, son unicité afin de se construire professionnellement.

\subsection{Charlotte et Claire : déliance, reliance dans le processus de construction identitaire professionnelle}

Après son stage en crèche, Charlotte considère que ce secteur ne lui convient pas. Elle éprouve un sentiment d'inutilité. II apparaît très vite pour elle un écart entre sa représentation du secteur de la petite enfance et l'activité réelle, autrement dit, entre l'activité éprouvée et ses aspirations. La dimension identitaire, dans le processus de négociation entre le soi et l'idéal de soi, (Dubar, 2007), est mobilisée. Le sujet ne souhaite ou ne peut se reconnaître dans l'offre identitaire qui lui est faite.

De la même façon, Claire exprime fortement sa déception, son ennui et le manque de réflexion de la part des professionnels au cours des stages en crèche et en halte d'enfants. Ce secteur lui semble fermé et porteur d'une vision réductrice de l'enfant. Elle vit cette expérience comme un désaccord entre ce qu'elle est (le Soi actuel) et ce à quoi elle aspire pour son propre développement (l'idéal de soi). Lorsqu'elle évoque le manque de réflexion des professionnels dans le secteur de la petite enfance, cet élément va dans le sens d'un désengagement de soi d'un espace où elle se trouve intégrée par la formation, mais dont elle refuse en partie l'appartenance (Barbier, 2006). Le processus de déliance et l'affirmation de soi sont à l'œuvre, par un double mouvement de reliance et de déliance à soi, à une communauté de pratiques et à ses idées, dans laquelle Charlotte et Claire ne se reconnaissent pas. Elles choisissent alors une autre voie.

Pour aller plus loin, nous voyons à travers les mots choisis l'expression d'une souffrance identitaire (Dubar, 2009). L'expérience des stages, auprès des jeunes enfants, conduit à une forte tension identitaire (Bourgeois, 2006) faisant apparaître les écarts entre leurs aspirations profondes et l'activité réelle. Les catégories d'analyse, les représentations professionnelles dont elles sont porteuses, se trouvent interrogées. La réorientation vers une autre formation est une manière de revendiquer leur identité professionnelle, porteuse de l'image qu'elles ont, ou souhaitent avoir pour elles-mêmes, et pour celle proposée à autrui.

L'expérience de Charlotte, auprès d'enfants handicapés, représente un temps de transition et d'ouverture. Elle se situe à la fois dans le secteur de la petite enfance et dans celui de l'éducation spécialisée. La problématique familiale mobilise son intérêt. Elle fait le pont entre une expérience présente et un projet en construction. Cette situation illustre la notion de transition qui permet au sujet de passer d'un état à un autre, de se mettre en mouvement pour s'engager dans un processus de transformation. Le sujet se transforme au fil de son activité et donne une direction à sa professionnalisation (Wittorski, 2009). Des points de référence vont lui permettre de réorienter et d'élaborer sa trajectoire identitaire.

Les stages en protection de l'enfance sont vécus comme source de bonheur et de nouveauté. Le travail avec les parents, des adultes à part entière, les conforte et développe leur intérêt pour ce champ d'activité. Charlotte découvre que le métier d'éducateur spécialisé est plus large dans les offres d'emploi proposées. En tant qu'éducatrice de jeunes enfants, elle ne pourra y accéder. Cette expérience est déterminante dans son orientation. Les individus au sein des sociétés contemporaines vivent dans un monde incertain et mouvant. La réalisation de soi est essentielle pour l'équilibre du sujet et cette offre plus large ajoute une forme d'anticipation face à l'incertitude (Wittorski, 2009).

Les sujets à travers le langage nous donnent à voir le plaisir identitaire éprouvé et l'équilibre trouvé (Barbier, 2006). Chacune ressent la cohérence entre ses aspirations, ses activités et son environnement.

Les stages réalisés en protection de l'enfance agissent comme des révélateurs. Les professionnelles rencontrées s'enthousias- 
ment et rencontrent d'autres professionnels ayant des convictions et des connaissances pointues. Ici, comme l'explique Jorro (2013), I'individu éprouve un sentiment d'une liberté à agir. II s'engage à partir de ses motivations internes. Devenir éducateur spécialisé devient alors une évidence. Ces éléments montrent le rôle joué par les communautés de pratiques (Bourgeois, 2006). Nous comprenons que la rencontre avec d'autres professionnels est alors déterminante. Le partage d'idées et l'identification de nouvelles pratiques professionnelles ouvrent des possibles dans la trajectoire identitaire. Le sujet se représente les bénéfices qu'il peut y trouver en terme de représentation de soi pour soi, de soi pour autrui et donc de continuité et de plaisir identitaire. Cette orientation soutient la cohérence entre les différentes composantes de son identité. Ainsi, la participation à de nouvelles communautés de pratiques conduit à la construction de nouvelles trajectoires identitaires. Les sujets font acte de reliance avec eux mêmes et avec autrui grâce à l'expérience vécue au sein de leurs nouvelles communautés de pratiques.

\subsection{Retour sur les trois hypothèses}

Les éléments recueillis et leur analyse nous permettent d'apporter quelques éléments de réponse.

L'expérience pratique est un facteur d'interrogation du projet professionnel initial. Elle est une préoccupation inhérente au processus de formation. Les moments de stage sont utilisés, vécus et rapportés à travers les quatre entretiens comme une expérience, comme une forme de vérification venant, ou non, valider l'orientation professionnelle initiale. Quelque soit le parcours et le champ professionnel choisi, ce processus est à l'œuvre. Le rôle joué par les communautés de pratiques apparaît de façon significative dans le processus construction-transformation de l'identité professionnelle. En effet, chaque expérience pratique conduit à interroger l'activité professionnelle, le public, la forme ou l'organisation du travail ainsi que les éléments vécus dans l'espace personnel. L'alternance, comme dispositif de formation, favorise la dimension réflexive et conduit le sujet à une délibération sur soi à travers les orientations de son projet.

La valorisation de soi à travers le champ de la petite enfance ou de l'éducation spécialisée est présente dans le discours de chacune des professionnelles rencontrées. Les deux champs sont source de valorisation et de réalisation de soi. Chaque sujet en formation, par l'expérience, par les rencontres, par les identifications ou adhésions possibles, se construit en cohérence avec son histoire, ses intérêts, ses découvertes. Chacune d'entre elles infléchit son choix, qu'il soit de réorientation ou bien de continuité du projet professionnel. La valorisation de soi à travers le choix de secteur grandit à la fois par la reconnaissance des professionnels qui les accueillent, et dans lesquelles elles se reconnaissent et par l'activité professionnelle. Cette valorisation s'appuie aussi sur des éléments liés à la vie personnelle.

La transformation de soi et la continuité de soi sont au centre du processus de professionnalisation et de construction identitaire. La formation vient modifier l'équilibre des différentes composantes de l'identité et les sujets en parcours de réorientation professionnelle sont particulièrement concernés. Car il s'agit pour eux de s'affirmer face à autrui, cet autrui pouvant se montrer opposé à ce projet. Cela engage le futur professionnel à se lier et à se délier dans le même espace-temps de formation. L'alternance produit à la fois une forme de déséquilibre pour le sujet et l'opportunité de sa transformation. C'est à ce moment que les tensions identitaires sont les plus vives. Le sujet, par une posture réflexive, se trouve en déséquilibre à ces moments de transition. Si les sujets en réorientation semblent davantage concernés, ce processus est aussi à l'œuvre pour les situations de continuité. Lorsque les personnes ne changent pas de voie professionnelle, elles accomplissent néanmoins toute une série de choix, de renoncements au regard de leurs représentations initiales. Elles délibèrent et construisent leur voie.

\section{Pour conclure}

Les résultats présentés font apparaître dans le processus de professionnalisation et de construction identitaire un construit de reliance engagé par les sujets en formation. Les dispositifs en alternance favorisent cette activité de reliance, de liance ou de déliance par les offres de sens faites à travers les rencontres avec les communautés de pratiques. L'alternance, comme dispositif de formation, amène les sujets, par sa dynamique réflexive, à des déplacements de point de vue, à des liens de chacun avec des éléments biographiques. Cela vient alors interroger le projet professionnel et conduit à une transformation identitaire personnelle et professionnelle.

Les premiers éléments issus de cette recherche nous invitent à être attentif à l'acte de reliance dans la formation en alternance des travailleurs sociaux. II révèle la singularité et l'activité du sujet en formation, au delà des dispositifs mêmes, dans une démarche de professionnalisation et de construction identitaire. La reliance, vécue et agie, engage les différentes composantes de l'identité et participe à l'élaboration du sens, au projet de soi dont le projet de formation est ici un élément central. 


\section{Références bibliographiques}

Arrêté du 16 novembre 2005 relatif au diplôme d'État d'éducateur de jeunes enfants. Téléacessible à: http://www.social-sante. gouv.fr

Astier, P. (2008). La professionnalisation comme intention, comme processus et comme légitimation. Savoirs, (17), 63-69.

Barbier, J.M., Bourgeois, E., Chapelle G. \& Ruano-Borbalan, J.C. (2009). Encyclopédie de l'éducation et de la formation. Paris: PUF.

Barbier, J.M. Bourgeois, E. de Villers, G., Kaddouri M. (2006). Constructions identitaires et mobilisation des sujets en formation. Paris, L'Harmattan.

Barbier, J. M. (2006). Problématique identitaire et engagement des sujets dans les activités. Dans J.M. Barbier, E. Bourgeois, G. de Villers \& M. Kaddouri, Constructions identitaires et mobilisation des sujets en formation (p. 15-64). Paris: L'Harmattan.

Bolle de Bal, M. (2003). Reliance, déliance, liance : émergence de trois notions sociologiques. Sociétés, (8)0, 99 -131. Téléacessible à: www.cairn.info/revue-societes-2003-2-page-99.htm.

Bourgeois, E. (2006). Tensions identitaires et engagement en formation. Dans J.M. Barbier, E. Bourgeois, G. de Villers \& M. Kaddouri, Constructions identitaires et mobilisation des sujets en formation (p. 65-120). Paris : L'Harmattan.

Bourgeois, E. (2013). Engagement et apprentissage en situation de travail dans les métiers de l'éducation et de la formation. Dans A. Jorro et J.M. De Ketele. L'engagement en éducation et formation (p.175-183). Bruxelles: De Boeck

Cohen-Scali, V. et Molinier P. (2008). Représentations sociales et identité : des relations complexes et multiples, Orientation Scolaire Professionnelle, 37 (4), 465-482.

Dubar, C. (2000). La crise des identités Paris : Presses Universitaires de France.

Dubar, C. (2007). Polyphonie et métamorphose de la notion d'identité. La documentation française, (2), 9-25.

Jorro A. \& De Ketele J.M. (2013). Défis professionnels et scientifiques de l'engagement en éducation et en formation. Dans A. Jorro et J.M. De Ketele. L'engagement en éducation et formation (p.165-173). Bruxelles: De Boeck.

Kaddouri, M. (2006). Dynamiques identitaires et rapports en formation. Dans J.M. Barbier, E. Bourgeois, G. De Villers, \& M. Kaddouri. Constructions identitaires et mobilisation des sujets en formation (p.121-145). Paris: L'Harmattan.

Le Moigne, J.L. (2008). Edgar Morin, le génie de la Reliance. Synergies Monde, (4), 177-184.

Neuville S., Van Dam S. (2006). Images de soi, motivation et engagement en formation. Dans J.M. Barbier, E. Bourgeois, G. de Villers et M. Kaddouri, Constructions identitaires et mobilisation des sujets en formation (p.15-64). Paris: L'Harmattan.

Perez-Roux, T. (2012) Introduction. Les Sciences de l'éducation - Pour l'Ėre nouvelle, 3 (45), 7-15. Téléacessible à : http://www. cairn.info/revue-les-sciences-de-l-education-pour-l-ere-nouvelle-2012-3-page-7.htm

Sebastian, C. \& de Villers G. (2006). Activité identitaire et sens de la formation. Dans J.M. Barbier, E. Bourgeois, G. de Villers \& M. Kaddouri. Constructions identitaires et mobilisation des sujets en formation (p.185-228). Paris: L'Harmattan.

Le Boterf, G. (2011). Apprendre à agir en professionnel compétent et responsable. Éducation Permanente, (188), 97-111.

Wittorski, R. (2005). Formation, travail et professionnalisation. Paris: L'Harmattan.

Wittorski, R. (2009). À propos de la professionnalisation. Dans J.M Barbier., E. Bourgeois, G. Chapelle \& J.C. Ruano-Borbalan, Encyclopédie de l'éducation et de la formation (p.781-793). Paris, France : Presses Universitaires de France.

Wittorski, R. (2011). Les rapports entre professionnalisation et formation. Éducation Permanente, (188), 5-9. 